\title{
Osteolytic orbital lesion: a case report
}

Chun-Wah Lam, ${ }^{1,2}$ MRCSEd (Ophth); Hunter KL Yuen, ${ }^{1,2}$ FRCS (Ophth); Wah Cheuk, ${ }^{3}$ FRCPA; Wai-Lun Poon, ${ }^{4}$

FHKAM (Radiology), FRCR

'Hong Kong Eye Hospital, Hong Kong SAR

${ }^{2}$ Department of Ophthalmology and Visual Sciences, Chinese University of Hong Kong, SAR

${ }^{3}$ Department of Pathology, Queen Elizabeth Hospital , Hong Kong SAR

${ }^{4}$ Department of Radiology and Imaging, Queen Elizabeth Hospital, Hong Kong SAR

Correspondence and reprint requests:

Dr Chun-Wah Lam, Hong Kong Eye Hospital, Hong Kong SAR.

Email: matthewlam100@hotmail.com

\section{Abstract}

A 62-year-old woman presented with optic neuropathy, proptosis, and ocular dysmotility secondary to an osteolytic orbital tumor. Subsequent tests confirmed the diagnosis of multiple myeloma. Differential diagnoses of osteolytic orbital lesions were discussed.

Key words: Multiple myeloma; Orbital neoplasms

\section{Case Presentation}

In April 2019, a 62-year-old Chinese woman presented with a 2-month history of progressive proptosis and reduced vision of the left eye. On examination, her visual acuity was vague light perception in the left eye and 6/6 in the right eye. The left eye had optic neuropathy with $5 \mathrm{~mm}$ of proptosis and impaired ocular motility in all directions. Fundal examination revealed relative afferent pupillary defect and gross disc swelling with choroidal folds in the left eye. Left facial sensation was reduced over the dermatomes V1 and V2.

Magnetic resonance imaging (MRI) of the brain and orbit demonstrated a 4.8-cm T1- and T2-weighted isointense lesion centered at the left lesser and greater wings of sphenoid bone with involvement of the left posterior orbit (Figure 1). It abutted the lateral rectus muscle and extended posteriorly to the anterior part of the left middle cranial fossa causing compression of the left temporal lobe. There was homogenous contrast enhancement after gadolinium contrast injection. Multiple enhancing skull vault lesions were also identified.

Systemic test results showed normochromic normocytic anemia and increased serum immunoglobulin $\mathrm{G}$ and Bence-

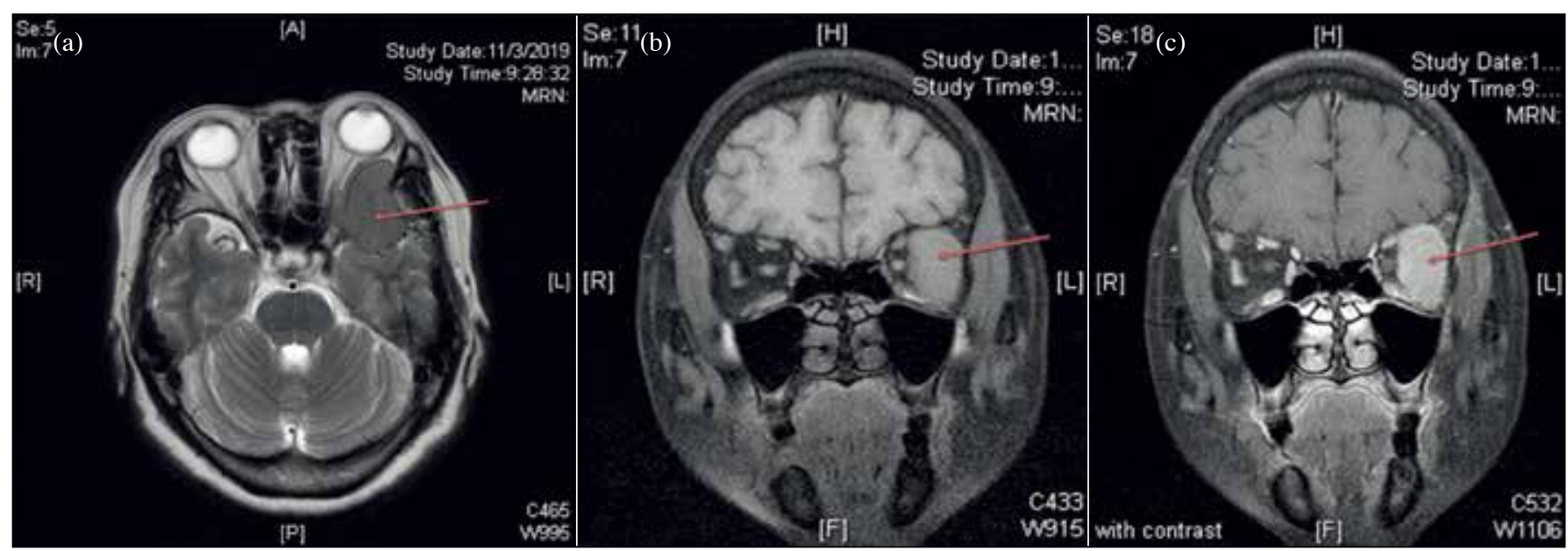

Figure 1. Magnetic resonance imaging showing a left orbital mass in (a) T2-weighted axial view, (b) T1-weighted coronal view, (c) and T1-weighted coronal view with homogeneous contrast enhancement 


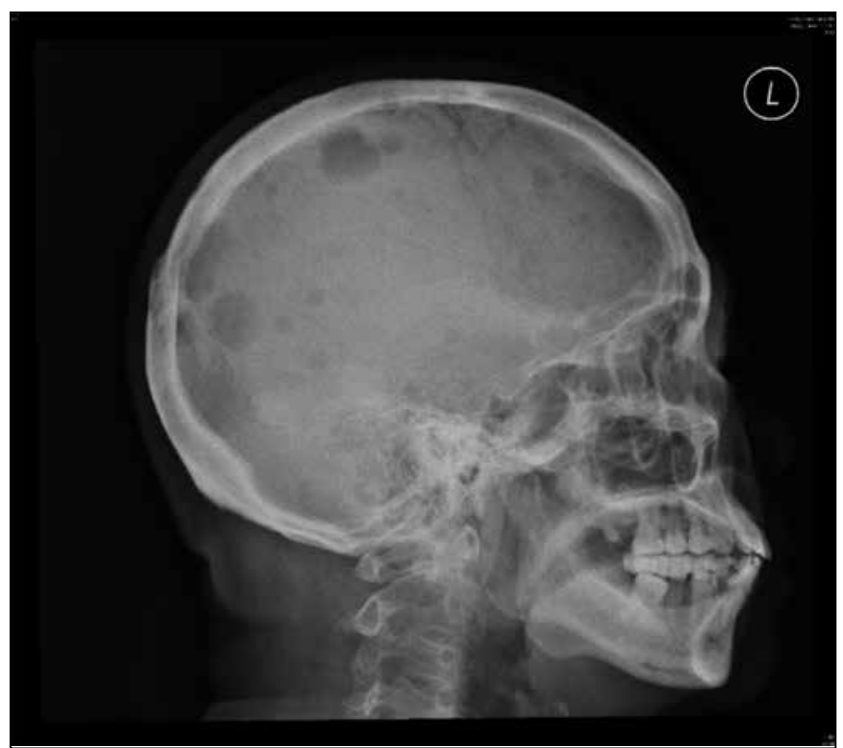

Figure 2. Skull radiograph showing multiple osteolytic lesions

Jones proteinuria. Skeletal examination revealed lytic lesions in multiple ribs, left humerus, and bilateral femurs. Lateral radiographs of the skull showed multiple osteolytic cranial lesions (Figure 2). Bone marrow biopsy revealed markedly hypercellular marrow with abnormal plasma cell infiltrate, and a diagnosis of multiple myeloma was made. Left anterior orbitotomy was performed with skin-crease incision, followed by incision of orbicularis muscle and orbital septum. Posterior soft tissue dissection enabled identification of the orbital mass. Biopsy of the lesion confirmed the involvement by myeloma. The abnormal plasma cells comprised eccentric nuclei, prominent Golgi zone, fine chromatin, and distinct nucleoli. The neoplastic plasma cells were positive for kappa light chain but negative for lambda light chain, indicative of clonal proliferation (Figure 3).

Chemotherapy was started and the left orbital mass and left eye proptosis reduced considerably. At the 17-month followup, the patient visual acuity was finger count in the left eye, with a pale optic disc.

\section{Discussion}

Multiple myeloma and orbital bone metastases are the most common differential diagnoses of osteolyticlesions of the orbit, especially when multiple lesions are detected systemically or when there is a known background of malignancy. Less common differential diagnoses include hyperparathyroidism, adenoid cystic carcinoma of the lacrimal gland, osteosarcoma, Ewing sarcoma, aneurysmal bone cyst, orbital sarcoidosis, and, rarely, intraosseous meningioma with osteolytic activity. Langerhans cell histiocytosis should also be borne in mind in the pediatric age group.

Orbital myeloma is uncommon and accounts for $3 \%$ of all orbital tumors. ${ }^{1}$ It is likely part of systemic multiple myeloma

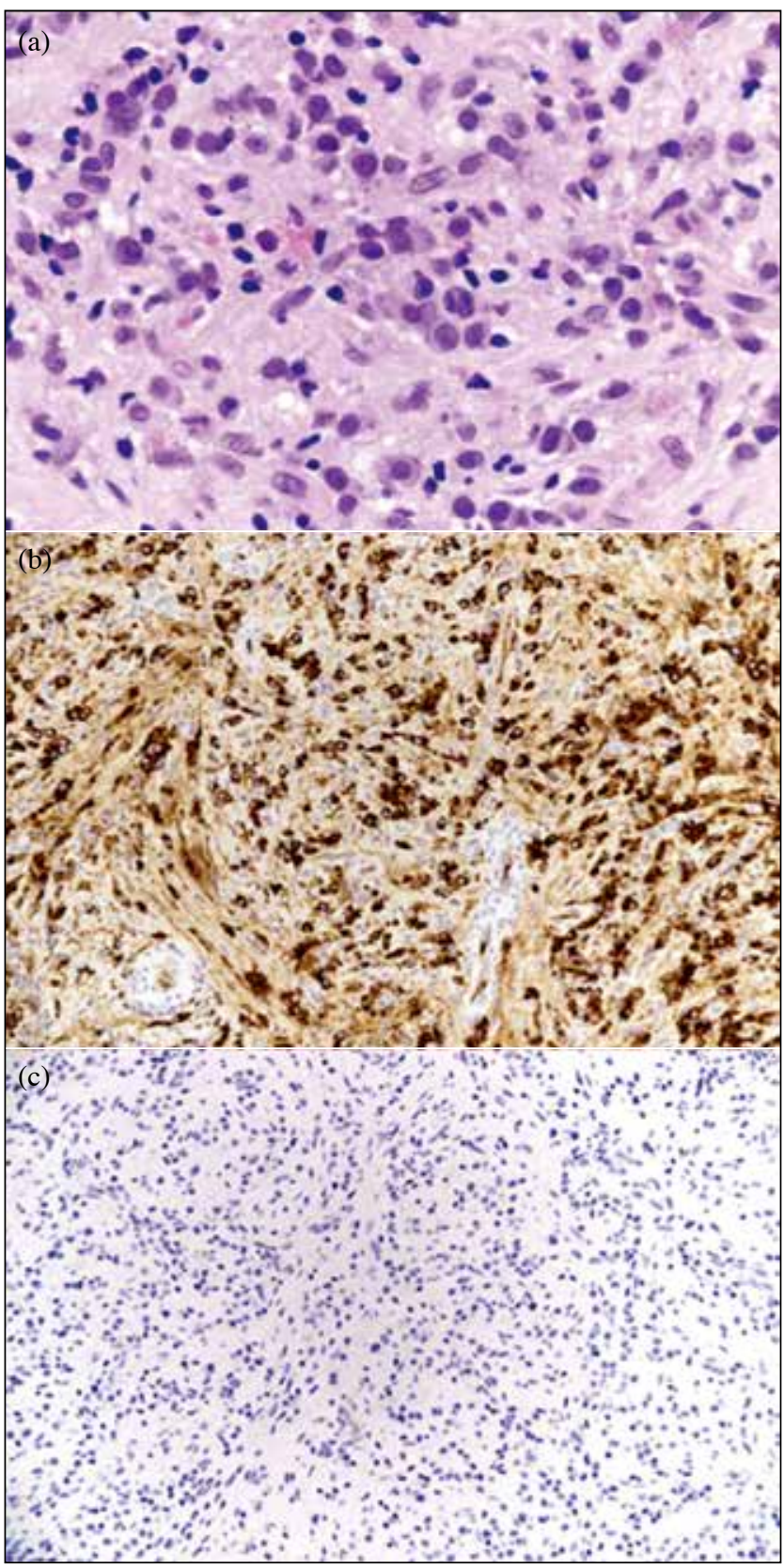

Figure 3. (a) The abnormal plasma cells comprise eccentric nuclei, prominent Golgi zone, fine chromatin, and distinct nucleoli. The neoplastic plasma cells are (b) positive for kappa light chain but (c) negative for lambda light chain, indicative of clonal proliferation.

rather than an isolated lesion. $60 \%$ of cases have known myeloma at the time of diagnosis, but the orbital lesion can precede multiple myeloma in more than one-third of patients. ${ }^{2}$ A solitary orbital lesion of clonal plasma cells is known as plasmacytoma; there is no end-organ damage or marrow involvement, in contrast to multiple myeloma.

Orbital myeloma most commonly occurs in the supratemporal quadrant of the orbit. It may be mistaken as a lacrimal gland tumor, especially in the absence of background systemic myeloma. Clinical features include proptosis, chemosis, 
ocular dysmotility, optic neuropathy, and choroidal folds. Occasionally, patients may have orbital cellulitis and hyperviscosity retinopathy, which are caused by the underlying hematological condition.

Treatment of orbital myeloma is chiefly chemotherapy followed by autologous stem cell transplantation. ${ }^{2}$ The role of surgery lies mainly in obtaining tissue for histological diagnosis. Radiotherapy may be useful for local disease control in selected cases. Multidisciplinary management is of utmost importance to arrive at an individualized treatment plan.

\section{Author contributions}

CWL was responsible for writing the draft manuscript and conducting the literature review. HKLY was involved in the management and performing biopsy of the patient. WC provided the pathology slides and figure captions. WLP selected the radiology images. All authors had full access to the data, contributed to the study, approved the final version for publication, and take responsibility for its accuracy and integrity.

\section{Conflicts of interest}

As an editor of the Journal, HKL Yuen was not involved in the peer review process for this article.

Other authors have disclosed no conflicts of interest.

\section{Funding/support}

This research received no specific grant from any funding agency in the public, commercial, or not-for-profit sectors.

\section{Ethics approval}

The patient was treated in accordance with the Declaration of Helsinki. The patient provided written informed consent for treatments and procedures.

\section{References}

1. Liao J, Greenberg A, Shinder R. Relapsed multiple myeloma presenting as an orbital plasmacytoma. Ophthalmic Plast

Reconstr Surg 2011;27:461. Crossref

2. Thuro BA, Sagiv $O$, Shinder $R$, et al.Clinical presentation and anatomical location of orbital plasmacytomas. Ophthalmic Plast Reconstr Surg 2018;34:258-261. Crossref

\section{Erratum}

In the article "Wong E, Chan A, Lam C, Lau W, Yam J, Yu C. Retinoblastoma in Hong Kong from 2008 to 2019: looking back and moving forward. Hong Kong J Ophthalmol 2020;24:6-10. https://doi.org/10.12809/hkjo-v24n1-270", a sentence in the results section of the abstract should have read "The enucleation rate was $0 \%$ in groups A to C, $70 \%$ in group D, and 93.3\% in group E." In addition, a sentence in the results section should have read "The rate of enucleation was proportionally related to the presenting stage ( $0 \%$ in groups A to C, $70 \%$ in group D, and $93.3 \%$ in group E)." 